\title{
Transcatheter closure of perimembranous ventricular septal defects with the Amplatzer asymmetric ventricular septal defect occluder: preliminary experience in children
}

\author{
B D Thanopoulos, G S Tsaousis, E Karanasios, N G Eleftherakis, C Paphitis
}

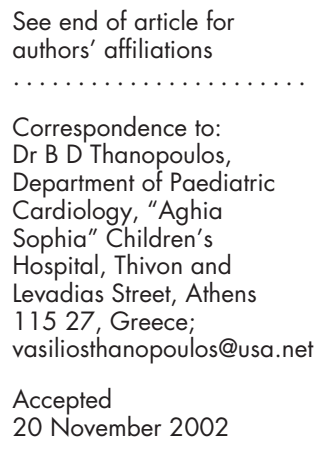

$20 \mathrm{November} 2002$

\begin{abstract}
Objective: To close perimembranous ventricular septal defects (PMVSDs) in children with the new Amplatzer asymmetric ventricular septal defect occluder (AAVSDO).

Patients and design: 10 children, aged 1.5-12 years, with PMVSDs underwent transcatheter closure with the AAVSDO. The device consists of two low profile disks made of Nitinol wire mesh with a $1.5 \mathrm{~mm}$ connecting waist. The left disk is $5 \mathrm{~mm}$ towards the apex and only $0.5 \mathrm{~mm}$ towards the aortic valve. The right disk is $4 \mathrm{~mm}$ larger than the waist. The prosthesis diameter was chosen to be 1-2 $\mathrm{mm}$ larger than the largest diameter of the defect (determined by transoesophageal echocardiography and angled angiocardiography). A 7-8 French gauge sheath was used to deliver the AAVSDO.

Results: The PMVSD diameter ranged from 2-8 $\mathrm{mm}$. The device diameter ranged from 4-8 $\mathrm{mm}$. After deployment of the prosthesis there was no residual shunt in 9 of 10 patients $(90 \%)$. In one patient there was a trivial residual shunt that disappeared at the three month follow up. Three patients developed transient complete left bundle branch block. No other complications were observed.

Conclusions: The AAVSDO appears to be a promising device for transcatheter closure of PMVSDs in children. Further studies are required to document its efficacy, safety, and long term results in a larger patient population.
\end{abstract}

I solated ventricular septal defect (VSD) is the most commonly recognised form of cardiac malformation and constitutes over $20 \%$ of all congenital cardiac disease. ${ }^{1}$ Eighty per cent of these defects are perimembranous involving the membranous septum and the adjacent area of muscular septum. Although conventional surgery for perimembranous VSDs (PMVSDs) is a widely accepted procedure with minimal operative mortality, it is associated with morbidity, postoperative patient discomfort, and a thoracotomy scar. ${ }^{1}$ Therefore, as an alternative approach to surgery, transcatheter closure of PMVSDs has been attempted using a variety of occluding devices. However, these devices were not specifically designed for this purpose and none has gained wide acceptance. Large delivery sheaths, inability to recapture and reposition, structural failure, dislodgement and embolisation of the device, interference with the aortic valve resulting in aortic insufficiency, and a high rate of residual shunting are the major limitations of the previously described techniques. ${ }^{2-7}$ We present our preliminary experience with transcatheter closure of PMVSDs in children with a new device that was especially designed for non-surgical occlusion of these defects, the Amplatzer asymmetric VSD occluder (AAVSDO). ${ }^{8}$ This device was recently redesigned and has been evaluated in in vivo experimental studies in animals with promising results that support initiation of clinical trials (K Amplatz, personal communication, 2001).

\section{METHODS}

\section{Device and delivery system}

The AAVSDO is a self centring and repositionable device. It consists of two low profile disks of Nitinol wire mesh (NDC, Fremont, California, USA) with a very short $(1.5 \mathrm{~mm})$ connecting waist to minimise contact with the tricuspid valve. In its latest design the left sided disk extends $5 \mathrm{~mm}$ towards the apex (with a platinum marker) to lean firmly on the muscular septum and only $0.5 \mathrm{~mm}$ towards the aorta to avoid contact with the aortic valve (fig 1). The right sided disk is $4 \mathrm{~mm}$ larger than the waist. The prosthesis is filled with Dacron fabric to facilitate thrombosis. Prostheses are available in sizes ranging from $4-12 \mathrm{~mm}$ at increments of $2 \mathrm{~mm}$. The device is attached by a microscrew mechanism on to a 0.038 inch delivery cable made of stainless steal. The delivery cable is placed within a delivery catheter with a curved tip to ensure alignment of the superior portion towards the aortic valve. A specifically designed metallic slot on the tip of the delivery catheter, which matches a flat superior area on the microscrew attachment, ensures alignment of the asymmetric aortic rim with the superior part of the curved tip of the delivery catheter (fig 2A) The delivery system is also curved to direct the tip of the sheath towards the left ventricular apex when across the VSD (fig 2B). In contrast to the other Amplatzer occluding devices the proximal part of the delivery cable goes all the way through the plastic vice (facilitating unscrewing of the device) to keep the aortic rim of the device in line with the superior curve of the tip of the delivery catheter during deployment of the left disk ( fig 2C). For introduction into the delivery sheath the device is pulled into a loader (fig 2D).

\section{Patients}

Between November 2001 and February 2002, 10 patients aged 1.5-12 years with PMVSDs underwent transcatheter closure with the AAVSDO (AGA Medical Corporation, Golden Valley, Minnesota, USA). In all patients VSD closure was indicated for haemodynamic or other medical reasons. All devices were implanted in the context of a research protocol approved by the ethical committee of "Aghia Sophia" Children's Hospital of Athens. Informed consent was obtained for each patient.

Abbreviations: AAVSDO, Amplatzer asymmetric ventricular septal defect occluder; PMVSD, perimembranous ventricular septal defect; VSD, ventricular septal defect 


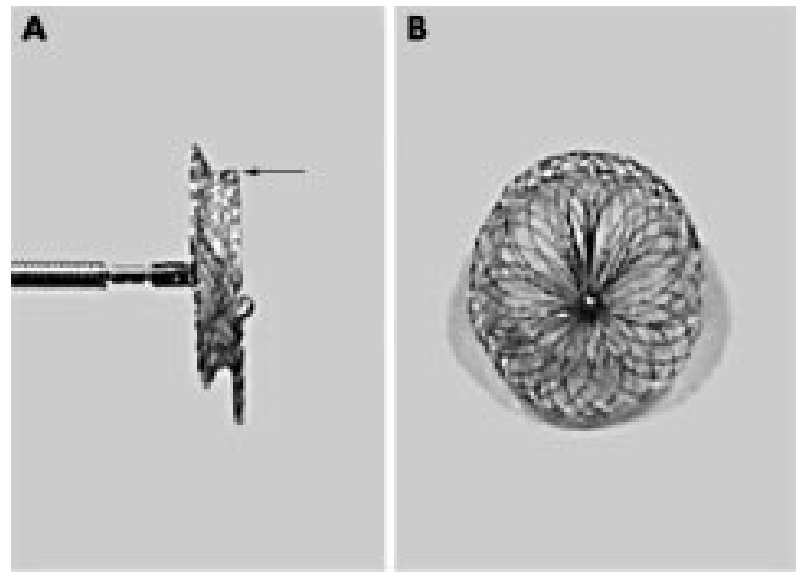

Figure 1 (A) Side and (B) frontal views of the Amplatzer asymmetric ventricular septal defect occluder (AAVSDO) made of woven Nitinol wires into two flat disks with a $1.5 \mathrm{~mm}$ connecting waist. Arrow indicates the $0.5 \mathrm{~mm}$ superior margin of the asymmetric left disk.

\section{Study design}

Patients were screened with transthoracic two dimensional and colour Doppler echocardiography in the subxiphoid, apical, and parasternal views. Patient selection criteria were maximal VSD diameter $<12 \mathrm{~mm}$, a distance of at least of $2 \mathrm{~mm}$ from the aortic valve, and left to right shunt with accompanying left ventricular enlargement.

\section{Procedure}

The technique of transcatheter closure of PMVSDs was similar to that developed by Lock and colleagues ${ }^{2}$ and modified by Thanopoulos and associates ${ }^{9}$ for muscular VSDs. The patients who met the selection criteria were intubated and underwent right and left heart catheterisation under general anaesthesia. After a complete saturation and haemodynamic study the location and size of PMVSDs and their relation to the surrounding structures were defined by transoesophageal echocardiography and angled angiographic views (fig $3 \mathrm{~A}$, fig $4 \mathrm{~A}$ ). In one patient it was difficult to measure the diameter of the VSD by left ventriculography and echocardiography. Therefore, the VSD was sized by balloon from the venous size using a balloon tipped end hole catheter (Arrow International, Reading, Pennsylvania, USA) that was advanced across the defect over the guidewire. In all patients the VSD was crossed from the left ventricle using a 4-5 French Cobra type I catheter (Cordis, Miami, Florida, USA). This was facilitated by small hand injections of contrast medium through a second arterial catheter (4-5 French Pigtail, Cordis) that was introduced from the opposite femoral artery. Through the cobra catheter a soft $0.035 \mathrm{inch}, 300 \mathrm{~cm}$ exchange guidewire (Ropewire, AGA Medical Corporation) was advanced into the pulmonary artery, where it was snared and withdrawn from the femoral vein establishing an arteriovenous loop. Then, the catheter was removed and a 7-8 French delivery sheath and dilator were advanced over the wire into the ascending aorta. The dilator was removed and the sheath tip was then placed into the ventricular apex by deflecting the guidewire towards the apex (fig 3B). The guidewire was removed and the sheath was carefully flushed. Occasionally, the placement of the sheath tip to the left ventricular apex was possible by deflecting the guidewire into the ventricular apex before advancing the sheath and dilator across the wire. The device size (waist diameter) was selected to be 1-2 $\mathrm{mm}$ larger than the largest measured diameter of the defect or equal to the balloon's "stretched" diameter. The device was screwed to the tip of the delivery cable and the microscrew attachment was pulled into the slot of the delivery catheter (fig $2 \mathrm{~A}, \mathrm{~B}$ ). Then, the plastic vice was tightened on to the proximal part of the delivery cable to keep the superior part of the delivery catheter in line with the aortic rim of the occluder (fig 2C). The device was collapsed into the loader (fig 2D) and advanced into the tip of the delivery sheath by pushing the delivery catheter. Under fluoroscopic and echocardiographic guidance the sheath was withdrawn to the left ventricular outflow tract far from the mitral valve apparatus. Then the left disk was deployed and pulled back gently against the septum, which was observed by both hand injections of contrast medium and echocardiographic monitoring. Under gentle tension on the sheath the right disk was deployed by advancing the delivery catheter. The device was released only when its position was optimal and interference with the aortic valve and tricuspid valve structures had been excluded by colour Doppler transoesophageal echocardiography. After release of the occluder both colour Doppler and selective ventriculography and aortography were performed to detect aortic or tricuspid valve regurgitation and residual shunts (fig 3C,D, fig 4B). All patients were discharged on the day after the procedure and taking aspirin $3-5 \mathrm{mg} / \mathrm{kg}$ daily for six months.

All patients were studied by chest radiography, ECG, and transthoracic colour Doppler echocardiography one and three
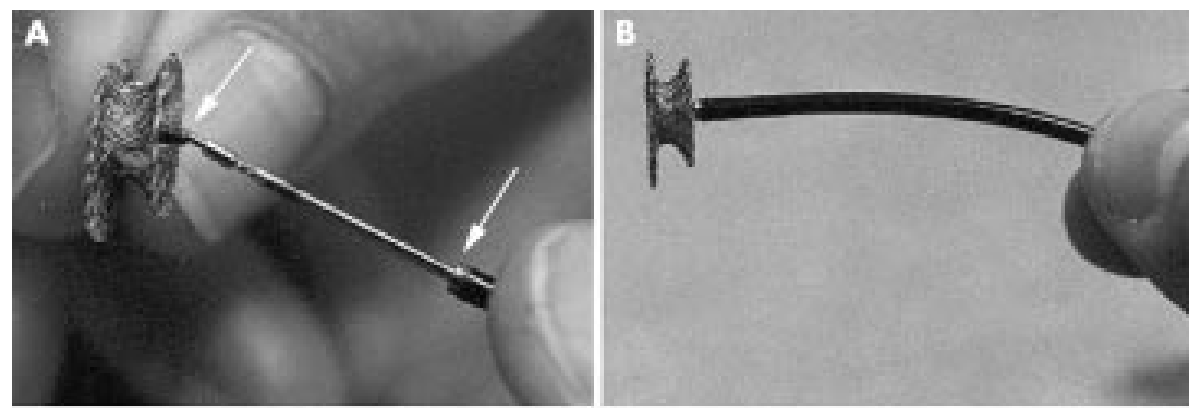

Figure 2 (A) Delivery system of the AAVSDO. Arrows indicate the negative and positive adaptors of the microscrew mechanism (upper arrow) and the metal slot (lower arrow) of the delivery catheter, respectively. (B) Side view showing an optimally loaded AAVSDO with its superior rim in line with the superior curve of the delivery catheter. (C) Tightening of the plastic vice on the delivery cable. (D) Loader used for introduction of the AAVSDO into the delivery sheath.
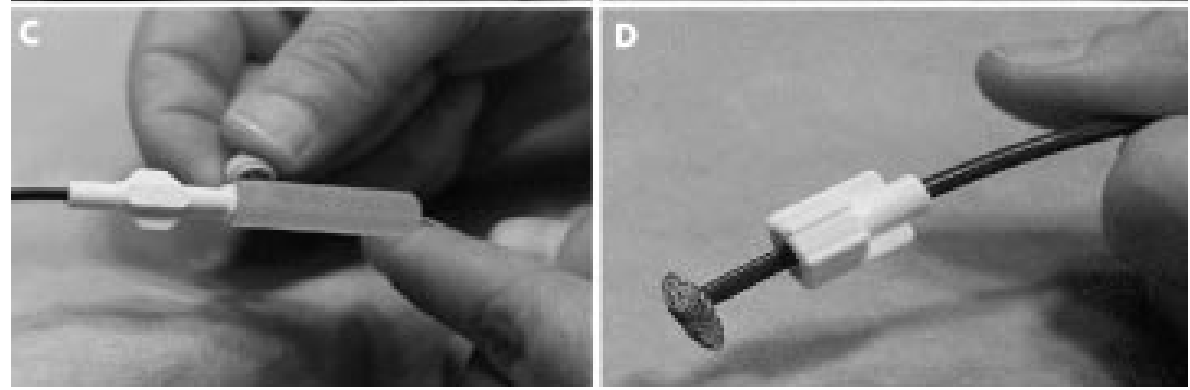

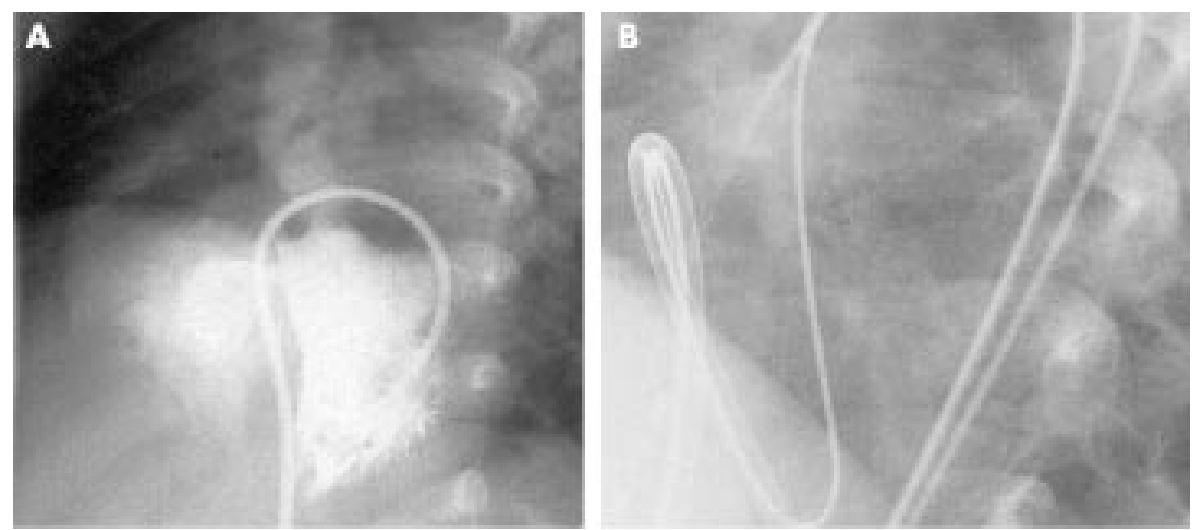

Figure 3 Steps in transcatheter perimembranous ventricular septal defect (PMVSD) closure with the AAVSDO. (A) Long axial oblique left ventriculogram obtained from patient 5, showing a PMVSD. (B) Cineradiographic frame showing sheath tip deflection to the left ventricular apex. (C) Long axial oblique ventriculogram after release of the AAVSDO showing complete closure with good device position.

(D) Ascending aortogram in the long axial oblique projection after the release of the device showing the absence of aortic regurgitation.
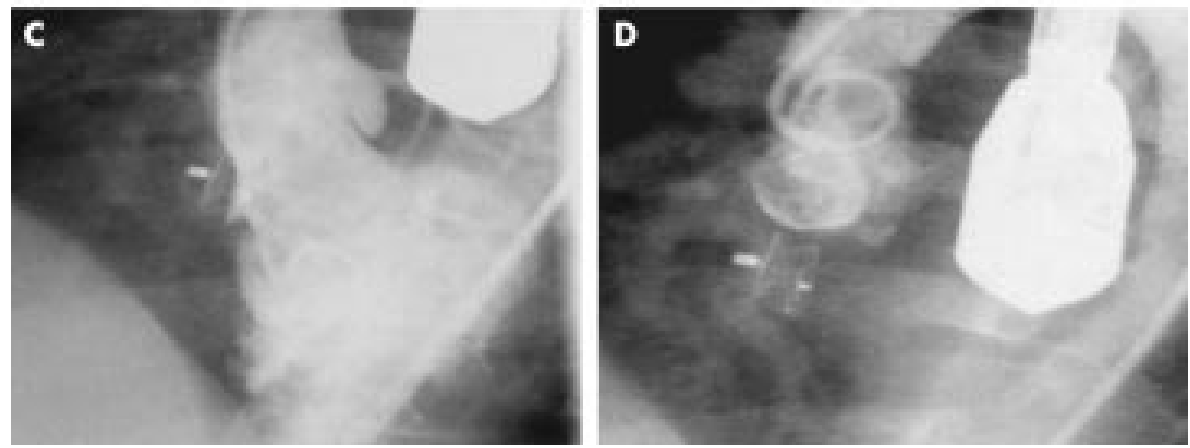

months later. Persistent shunts were angiographically and echocardiographically graded as foaming, trivial, small, moderate, and large as previously described..$^{10}$

\section{Statistical analysis}

Results are expressed as mean (SD), with confidence intervals given where applicable.

\section{RESULTS}

Table 1 presents descriptive statistics and outcome for all patients. The largest VSD diameter ranged from $2-8 \mathrm{~mm}$ (mean (SD) $4.5(1.9) \mathrm{mm}$ ). The device size ranged from
4-8 mm (mean (SD) 6 ( 1.6 ) mm). Pulmonary to systemic flow ratio varied from 1.5-2.4 (mean (SD) $1.9(0.3))$. Systolic pulmonary artery pressures were normal $(<40 \mathrm{~mm} \mathrm{Hg})$ in all patients. The ventricular septal rims below the aortic valve ranged from $2-5 \mathrm{~mm}$. Four patients had an associated small ventricular septal aneurysm. Patients 2 and 6 had a small VSD (pulmonary to systemic flow ratios 1.6 and 1.5, respectively) and history of two episodes of subacute infective endocarditis. Surgical VSD closure was suggested after the second episode but the parents refused it.

The right ventricular disk was misplaced in the left ventricle in one patient. This was successfully managed by recapturing
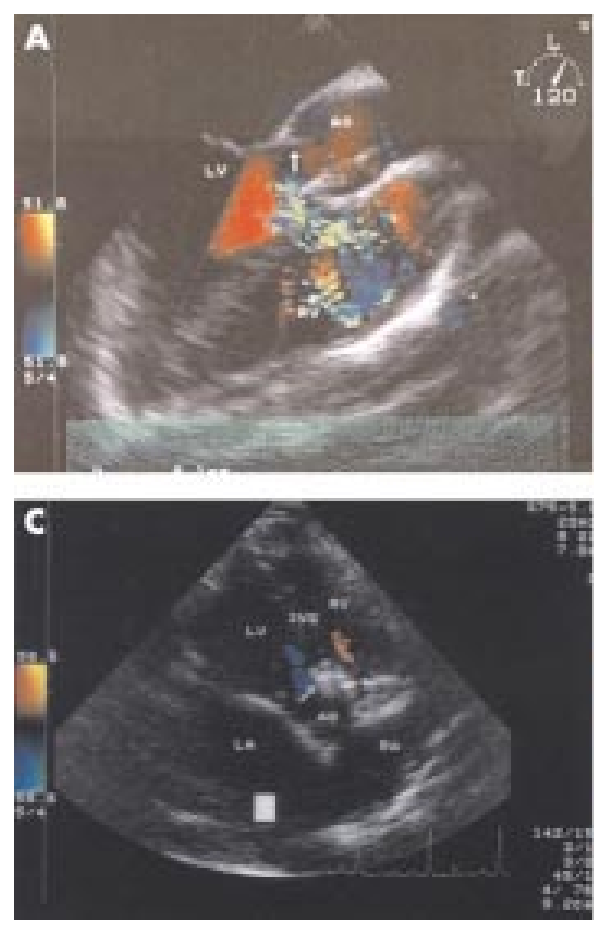

Figure 4 (A, B) Transoesophageal two dimensional and colour Doppler images of the membranous ventricular septum obtained from long axis and short axis views, respectively,

(A) before and (B) immediately after implantation of the AAVSDO in patient 8 . Note good position of the device (arrows) with no evidence of residual shunt. LV, left ventricle; RV, right ventricle. Arrow in $(\mathrm{A})$ indicates the level of the aortic valve. (C, D) Transthoracic two dimensional and colour Doppler images obtained in (C) systole and (D) diastole from the five chamber view three months after implantation of the AAVSDO.

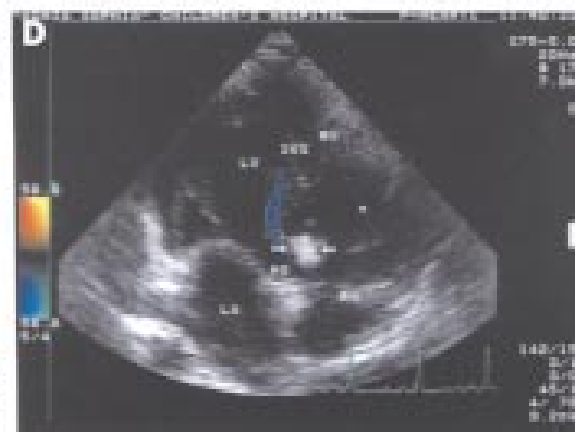
The device is in the appropriate position (arrows) and no residual shunt or aortic regurgitation is observed. AO, ascending aorta; IVS, interventricular septum; LA, left atrium; RA, right atrium. 
Table 1 Clinical and haemodynamic data in 10 patients with membranous ventricular septal defect (VSD)

\begin{tabular}{|c|c|c|c|c|c|c|c|c|c|c|}
\hline \multirow[b]{2}{*}{$\begin{array}{l}\text { Patient } \\
\text { number }\end{array}$} & \multirow[b]{2}{*}{$\begin{array}{l}\text { Age } \\
\text { (years) }\end{array}$} & \multirow[b]{2}{*}{ Weight (kg) } & \multirow[b]{2}{*}{$Q p / Q s$} & \multirow[b]{2}{*}{$\begin{array}{l}\text { VSD diameter } \\
(\mathrm{mm})\end{array}$} & \multirow[b]{2}{*}{$\begin{array}{l}\text { Device diameter } \\
(\mathrm{mm})\end{array}$} & \multirow[b]{2}{*}{ Complication } & \multicolumn{4}{|l|}{ Outcome } \\
\hline & & & & & & & $\begin{array}{l}\text { Immediate } \\
\text { angiogram }\end{array}$ & $\begin{array}{l}24 \mathrm{~h} \\
\mathrm{TTE}\end{array}$ & $\begin{array}{l}1 \text { month } \\
\text { TTE }\end{array}$ & $\begin{array}{l}3 \text { month } \\
\text { TTE }\end{array}$ \\
\hline 1 & 4 & 18 & 1.7 & 3 & 4 & TCLBBB & $\mathrm{CC}$ & $\mathrm{CC}$ & $\mathrm{CC}$ & $\mathrm{CC}$ \\
\hline 2 & 6 & 24 & 1.6 & 2.5 & 4 & None & $\mathrm{CC}$ & $\mathrm{CC}$ & $\mathrm{CC}$ & $\mathrm{CC}$ \\
\hline 3 & 1.5 & 11 & 2.2 & 4.5 & 6 & None & $\mathrm{CC}$ & $\mathrm{CC}$ & $\mathrm{CC}$ & $\mathrm{CC}$ \\
\hline 4 & 2 & 16 & 2.4 & 6 & 8 & TCLBBB & CC & $\mathrm{CC}$ & $\mathrm{CC}$ & CC \\
\hline 5 & 5 & 22 & 2.0 & 4 & 6 & None & $\mathrm{CC}$ & $\mathrm{CC}$ & $\mathrm{CC}$ & $\mathrm{CC}$ \\
\hline \multirow[t]{2}{*}{6} & 12 & 41 & 1.5 & 2 & 4 & None & $\mathrm{CC}$ & $\mathrm{CC}$ & $\mathrm{CC}$ & CC \\
\hline & & & & & & & $\mathrm{CC}$ & $\mathrm{CC}$ & CC & CC \\
\hline 8 & 4 & 20 & 2.3 & 7 & 8 & None & $\mathrm{CC}$ & CC & $\mathrm{CC}$ & $\mathrm{CC}$ \\
\hline 9 & 3 & 17 & 2.3 & $8 *$ & 8 & TCLBBB & TS & TS & TS & $\mathrm{CC}$ \\
\hline 10 & 12 & 49 & 1.9 & 6 & 6 & None & $\mathrm{CC}$ & $\mathrm{CC}$ & $\mathrm{CC}$ & $\mathrm{CC}$ \\
\hline Mean(SD) & $6.0(4.0)$ & $25.1(12.1)$ & $1.93(0.29)$ & $4.5(1.94)$ & $6.0(1.63)$ & & & & & \\
\hline
\end{tabular}

*Balloon "stretched" diameter.

CC, complete closure; $\mathrm{Qp} / \mathrm{Qs}$, pulmonary to systemic flow ratio; TCLBBB, transient complete left bundle branch block; TS, trivial residual shunt; TTE, transthoracic echocardiography.

the device and redeploying it. In two small patients (patients 3 and 4) it was not possible to place the sheath tip to the left ventricular apex before advancing the device. In these patients a left ventricular outflow tract position was used for deployment of the left ventricular disk of the device. Immediately after implantation the device marker was between the 5 and $6.30 \mathrm{o}^{\prime}$ clock positions (mean $5.30 \mathrm{o}^{\prime}$ clock) of an analogue clock (long axial oblique view). After release of the device left ventriculography and colour Doppler echocardiography showed no residual shunt in 9 of the 10 patients (90\% closure rate, $95 \%$ confidence interval $55.50 \%$ to $99.75 \%$ ). One patient had a trivial residual shunt immediately after the procedure. Foaming (trace angiographic residual shunt and no contrast jet) was present in three patients but disappeared within 15-20 minutes. No patient developed aortic regurgitation. Four patients had a trivial to mild tricuspid regurgitation, which did not progress after the procedure. During the procedure three patients developed transient complete left bundle branch block. No other complications were observed. Fluoroscopy and total procedure times ranged from 38-59 minutes (mean 44 minutes) and from 118-158 minutes (mean 146 minutes), respectively.

Follow up echocardiographic data were available in all 10 patients at one and three months after the procedure. There was no evidence of residual shunts (100\%, 95\% confidence interval $69.15 \%$ to $100 \%$ ). The device was in an appropriate position and no interference with the adjacent cardiac structures was observed (fig 4C,D). Metal fatigue fractures on chest radiography were not observed. There was no evidence of device migration, conduction defects on ECG, thromboembolism, endocarditis, or haemolysis.

\section{DISCUSSION}

In 1988 Lock and colleagues developed an elaborate technique for transcatheter closure of muscular VSDs and residual PMVSD after cardiac surgery using initially the Rashkind double umbrella and later the clamshell device. ${ }^{23}$ Subsequently the Rashkind double umbrella ${ }^{4}{ }^{6}$ and the Sideris buttoned device ${ }^{7}$ have been used for transcatheter closure of PMVSDs, but they have not met their expectations. The Rashkind double umbrella was specifically designed for closure of a persistent arterial duct. The clamshell occluder and the Sideris buttoned device were designed for occlusion of secundum atrial septal defects. Therefore, their shape and design are not ideal for transcatheter closure of PMVSDs. The major drawbacks of these devices are large delivery sheaths limiting their use in infants and small children, complex implantation techniques, inability to reposition and redeploy, interference with the aortic and tricuspid valves, and significant residual shunts. ${ }^{4-7}$
There is only one published report in the literature of transcatheter closure of PMVSDs with very good initial results. ${ }^{11}$ In our study we used the AAVSDO for transcatheter closure of PMVSDS in 10 patients. Complete occlusion was achieved in all patients with no complications during the procedure or at short term follow up. It appears therefore that the efficacy and safety of the procedure are predominantly related to deployment technique and the novel design of the occluder.

\section{Comparison with other devices}

The AAVSDO is a specifically designed device for occlusion of PMVSDs, which offers significant advantages over the previously used devices. Besides the Sideris device, which is delivered through a 7-9 French sheath, all other occluders are delivered through an 11 French sheath, which make their application difficult or impossible in small children. In contrast to the other occluders that accomplish closure of the VSD by the retention flanges (patches) the AAVSDO uses its short connecting waist to stent the communication, forcing blood to flow through a network of highly thrombogenic polyester material. Therefore, closure by thrombosis should be virtually $100 \%$. The previously reported incidence of residual shunting $(25-60 \%)$ is much higher than in the present study. ${ }^{4-6}$ A very important property of the AAVSDO is the asymmetry of the left ventricular disk. The asymmetric left ventricular disk, as well as the loading system, was especially designed to avoid contact with the aortic valve and to ensure stability of the device across the defect. In contrast to the AAVSDO (which requires a $2 \mathrm{~mm}$ rim of tissue between the defect and the aortic valve), all other devices require a long distance (at least 6-8 $\mathrm{mm}$ ) between the aortic valve and the VSD, which limits their use in these types of defects. ${ }^{4-6}$ An additional advantage of the AAVSDO is the two round disks. Occluders with corners or spokes may cause accidental injury of aortic or atrioventricular valves during disk deployment. Also, delayed perforations may occur. Furthermore, complications previously reported, such as metal fatigue fractures, embolisations of the device, and aortic or atrioventricular valve damage, were not encountered in the present study, although the follow up was short. ${ }^{4-7}$

Two patients in this study with small defects and a history of subacute infective endocarditis underwent transcatheter device closure for prevention of further episodes of the infection. Although patients with small VSDs have an excellent prognosis, they are at risk for endocarditis and late cardiac arrhythmias. ${ }^{12}$ It is expected that complete closure of these defects will obviate the risk of endocarditis and arrhythmias and ensure a normal life span. ${ }^{6}$

Although transoesophageal and colour Doppler echocardiography play an important part in monitoring transcatheter 
device closure of cardiac defects, in our experience, for the closure of VSDs, this modality should always be combined with fluoroscopic guidance. ${ }^{9}$ A repeated left ventricular injection of a small amount of contrast medium through the arterial catheter greatly facilitates the guidance of the procedure and reduces the risk of device misplacement and embolisation. This is particularly useful in monitoring transcatheter closure of PMVSDs because in the case of such defects it is easy to misplace the occluder in the right ventricle. This is because during engagement of the left disk against the rims of the VSD the operator does not feel any resistance, as the membranous septum is very thin.

According to our preliminary experience a device size 1-2 mm larger than the maximal VSD diameter is appropriate for all PMVSDs including those with an associated small ventricular septal aneurysm. However, in the presence of larger septal aneurysms an oversized device should be used to keep the left disc of the AAVSDO in alignment with the muscular septum.

\section{Limitations of the study}

In this study we successfully closed PMVSDs (sized up to $8 \mathrm{~mm}$ ) in children from 1.5-12 years of age by using the AAVSDO. The application of this technique in infants and larger VSDs remains to be determined. Implantation of the AAVSDO is more complex and difficult than deployment of other Amplatzer devices including the Amplatzer muscular VSD occluder. ${ }^{9}{ }^{13}$ It is tedious and not always possible to place the sheath in the left ventricular apex in preparation for proper device deployment in small children. Modifications in the design of the delivery sheath and dilator to simplify proper sheath positioning are needed to eliminate this drawback and to facilitate the use of the AAVSDO in small paediatric patients.

\section{Conclusions}

Our preliminary results indicate that the AAVSDO is a promising device for transcatheter closure of PMVSDs in children. Further studies are required to establish long term results in a larger patient population.

\section{Authors' affiliations}

B D Thanopoulos, G S Tsaousis, E Karanasios, N G Eleftherakis,

C Paphitis, Department of Paediatric Cardiology, "Aghia Sophia"

Children's Hospital, Athens, Greece

There were no sources of financial support

Presented at the 37th Annual General Meeting of The Association for European Paediatric Cardiology, Porto, 15-18 May 2002

\section{REFERENCES}

1 Mavroudis C, Baker CL, Idriss FS. Ventricular septal defect. In: Mavroudis C, Baker CL, eds. Pediatric cardiac surgery, 2nd ed. St Louis: Mosby, 1994:201-24.

2 Lock JE, Block PC, McKay RG, et al. Transcatheter closure of ventricular septal defects. Circulation 1998;78:361-8.

3 Bridges ND, Perry SB, Keane JF, et al. Preoperative transcatheter closure of congenital muscular ventricular septal defects. N Engl J Med 1991:324:1312-7.

4 Rigby ML, Redington N. Primary transcatheter closure of perimembranous ventricular septal defect. Br Heart J 1994;72:368-71.

5 Vogel M, Rigby ML, Shore D. Perforation of the aortic valve cusp: complication of ventricular septal defect closure with a modified Rashkind umbrella. Pediatr Cardiol 1996;17:416-8.

6 Kalra GS, Verma PK, Dhall A, et al. Transcatheter device closure of perimembranous septal defects: immediate results and intermediate-term follow-up. Am Heart J 1999;138:339-44.

7 Sideris EB, Walsh KP, Haddad JL, et al. Occlusion of congenital ventricular septal defects by the buttoned device. Heart 1997;77:276-9.

8 Gu X, Han Y-M, Titus JL, et al. Transcatheter closure of membranous ventricular septal defects with a Nitinol prosthesis in a natural swine model. Cathet Cardiovasc Interv 2000;50:502-9.

9 Thanopoulos BD, Tsaousis GS, Konstadopoulou GN, et al. Transcatheterclosure of muscular ventricular septal defects with the Amplatzer ventricular septal defect occluder: initial clinical applications in children. J Am Coll Cardiol 1999;33:1395-9.

10 Thanopoulos BD, Laskari CV, Tsaousis GS, et al.Closure of atrial septal defects with the Amplatzer occlusion device: preliminary results. J Am Coll Cardiol 1998;31:110-6.

11 Hijazi Z, Hakim F, Havaleh A, et al. Catheter closure of perimembranous ventricular septal defects using the new Amplatzer membranous VSD occluder: initial clinical experience. Cathet Cardiovasc Interv 2002:56:508-15.

12 Kidd L, Driscoll DJ, Gersony WM, et al. Second natural history of congenital heart defects: results of treatment of patients with ventricular septal defects. Circulation 1993;87(suppl I):38-51

13 Hijazi ZM, Hakim F, Al-Fadley F, et al. Transcatheter closure of single muscular ventricular septal defects using the Amplatzer muscular VSD occluder: initial results and technical considerations. Cathet Cardiovasc Interv 2000;49: 167-7.

\section{www.heartjnl.com}

